\title{
Stochastic compartmental model of HIV-1 infection
}

\author{
Konstantin Loginov ${ }^{1, *}$ and Nikolai Pertsev ${ }^{1, * *}$ \\ ${ }^{1}$ Sobolev Institute of Mathematics, Siberian Branch of RAS, Omsk Department; 13 Pevtsova avenue, \\ 644043, Omsk, Russia.
}

\begin{abstract}
Stochastic model of the dynamics of HIV-1 infection describing the interaction of target cells and viral particles in the lymphatic nodes and their movement between the lymphatic nodes is constructed. The lymphatic system is represented as a graph, vertices of which are the lymphatic nodes and edges are the lymphatic vessels. The novelty of the model consists in the description of populations of cells and viral particles in terms of a multidimensional birth and death process with the random point-distributions. The random pointdistributions describe the duration of the transition of cells and viral particles between the lymph nodes and the duration of the stages of their development. The durations of transitions of viral particles and cells between the lymphatic nodes are not random and based on the rate of lymph flow. The durations of the developmental stages of infected target cells are assume to be constant. The graph theory for the formalization and compact representation of the model is used. An algorithm for modelling the dynamics of the studied populations is constructed basing on the Monte-Carlo method. The results of computational experiments for a system consisting of five lymphatic nodes are presented.
\end{abstract}

\section{Introduction}

An important direction in mathematical modeling in immunology is the development of mathematical models of the dynamics of HIV-1 infection in the human lymphatic system [1,2]. Mostly, these models are divided into deterministic and stochastic one. Deterministic models take the form of the systems of delay differential equations [3, 4]. The equations of such models describe concentration of target cells, infected cells, viral particles, etc. An example of a stochastic model is constructed at [5] in the form of multidimensional random birth and death process. A stochastic model with integer variables describing the dynamics of HIV-1 infection in a single lymph node in the form of random process with particle interaction is proposed at [6]. The lymphatic system is a collection of lymph nodes interconnected by lymphatic vessels through which cells and viral particles can move. Modelling movements of cells in the lymphatic system is needed the construction of a stochastic compartment model with pipes or its deterministic analogue. One of the first deterministic models in this direction presented at [7]. An important feature of the model described at [7] is the presence of transport delays, which reflect the duration of particle movement through the pipes. Other models of the lymphatic system based on partial differential equations including the NavierStokes equations are presented, for example at [8]. Mathematical model of the dynamics of

\footnotetext{
*e-mail: kloginov85@mail.ru

**e-mail: homlab@ya.ru
} 
HIV-1 infection in the form of high-dimensional system of ordinary differential equations is presented at [9]. The model described at [9] contains detailed information regarding the structure of the human lymphatic system, however, there are no transfer delays in the model equations. It is important to note that the absence of transport delays leads to the possibility of instantaneous and simultaneous changes in the number of populations in various lymph nodes caused by processes occurring in only one specific lymph node. Stochastic model with one type of particles moving in a compartment system with pipes is considered at [10].

The purpose of this article is to build a stochastic compartmental model of the dynamics of HIV-1 infection which combines the approaches proposed at $[6,10]$.

\section{Assumptions on the dynamics of HIV-1 infection}

In describing the dynamics of HIV-1 infection, we will use the following notation:

- $T$ denotes T-lymphocytes (target cells for mature viral particles);

- $C$ is infected cells, i.e. cells in preparation for the production of viral particles;

- $I$ is productively infected cells (cells producing viral particles);

- $U$ denotes immature viral particles;

- $V$ corresponds to mature viral particles (or virions);

- $K$ is precursor cells of effector lymphocytes;

- $E$ is related to cytotoxic T-lymphocytes (effectors).

Moreover, let $S$ denotes the cell source in bone marrow and lymphoid tissue generating the target cells $T$ and cells $K ; D$ is all dead cells and viral particles.

We will describe the lymphatic system in the form of graph G. The notations are as follows:

- $G$ is oriented graph without loops with weighted edges;

- $n$ is number of graph vertices $G$;

- $N_{i}$ denotes vertices of the graph $G$ (lymph nodes),

$1 \leqslant i \leqslant n$;

- $N_{i j}$ corresponds edges of the graph $G$ (lymph vessels), $1 \leqslant i, j \leqslant n, i \neq j$;

- $Q=\left\|q_{i j}\right\|$ is matrix defining relationships between vertices of the graph $G$; constants $0 \leqslant$ $q_{i j} \leqslant 1$ for all $i=1, \ldots, n$ satisfy the conditions:

$$
q_{i i}=0 ; \quad q_{i j} \cdot q_{j i}=0,1 \leqslant j \leqslant n, j \neq i ; \sum_{j=1}^{n} q_{i j}=1 .
$$

If $q_{i j}>0$, then vertices $N_{i}$ and $N_{j}$ interconnected by edge $N_{i j}$ but connection between $N_{j}$ and $N_{i}$ is absent. For $q_{i j}=0$ there is no connection between $N_{i}$ and $N_{j}$ but there may be a connection between $N_{j}$ and $N_{i}$.

- $\Delta_{i j}(t)$ is positive and bounded above real-valued function that defines the weight of the edge $N_{i j}, t \in(-\infty ; \infty), q_{i j}>0$. We assume that $\Delta_{i j}(t)+t$ is strictly increasing.

Example of the graph $G$ is presented on the figure 1 .

Denote

- $T_{i i}, C_{i i}, I_{i i}, U_{i i}, V_{i i}, K_{i i}, E_{i i}$ are components of the immune system and HIV-1 particles located in the vertex $N_{i}, 1 \leqslant i \leqslant n$; 


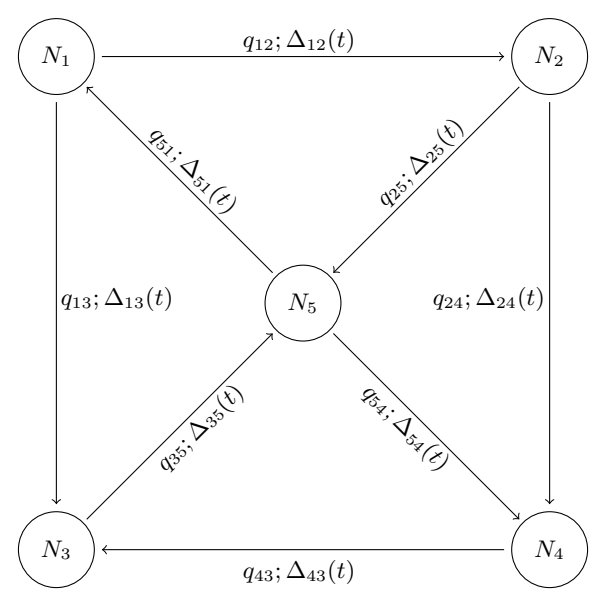

Figure 1. Example of the graph $G$ with five vertices

- $T_{i j}, C_{i j}, I_{i j}, U_{i j}, V_{i j}, K_{i j}, E_{i j}$ are components of the immune system and HIV-1 particles in the state of transition from vertex $N_{i}$ to vertex $N_{j}$ along edge $N_{i j}, 1 \leqslant i, j \leqslant n, i \neq j$.

Following $[2,4,6,10]$, we introduce the set of assumptions that determine dynamics of HIV-1 infection:

- Inflow of target cells $T$ to vertex $N_{i}$ with rate $r_{i i}^{(T)}>0: S \stackrel{r_{i i}^{(T)}}{\longrightarrow} S+T_{i i}$.

- Natural death of cells $T, C, I, E$ and particles $U, V$ :

$$
\begin{gathered}
T_{i i} \stackrel{\mu_{i i}^{(T)}}{\longrightarrow} D ; C_{i i} \stackrel{\mu_{i i}^{(C)}}{\longrightarrow} D ; I_{i i} \stackrel{\mu_{i i}^{(I)}}{\longrightarrow} D ; E_{i i} \stackrel{\mu_{i i}^{(E)}}{\longrightarrow} D ; \\
U_{i i} \stackrel{\mu_{i i}^{(U)}}{\longrightarrow} D ; V_{i i} \stackrel{\mu_{i i}^{(V)}}{\longrightarrow} D .
\end{gathered}
$$

- Interaction of $T$-cells with virions $V$ (cell infection) with rate $\gamma_{i i}^{(T, V)}>0$ per one pair of $(T, V): T_{i i}+V_{i i} \stackrel{\gamma_{i i}^{(T, V)}}{\longrightarrow} C_{i i}$.

- Infection of cells $T$ as a result of interaction with productively infected cells $I$ with rate $\gamma_{i i}^{(T, I)}>0$ per one pair of $(T, I): T_{i i}+I_{i i} \stackrel{\gamma_{i i}^{(T, I)}}{\longrightarrow} C_{i i}+I_{i i}$.

- Production by cells $I$ of immature viral particles of $U$ with rate $v_{i i}^{(U)}>0: I_{i i} \stackrel{v_{i i}^{(U)}}{\rightarrow} I_{i i}+U_{i i}$.

- Destruction of infected cells $I$ by cytotoxic cells $E$ with rate $\gamma_{i i}^{(I, E)}>0$ per one pair of $(I, E)$ : $I_{i i}+E_{i i} \stackrel{\gamma_{i i}^{(I, E)}}{\longrightarrow} D+E_{i i}$.

- Cell death $I$ due to the destructive process of production of viral particles with rate $\sigma_{U} v_{i i}^{(U)}$, $\sigma_{U}>0: I_{i i} \stackrel{\sigma_{U} v_{i i}^{(U)}}{\longrightarrow} D$.

- Formation of precursor cells $K$ cytotoxic lymphocytes $E$ with rate $v_{i i}^{(K)}>0: I_{i i} \stackrel{v_{i i}^{(K)}}{\longrightarrow} I_{i i}+K_{i i}$.

- The cell $C$ which appeared at time $t$ and did not die during the time interval $\left(t, t+\omega_{C}\right)$, $\omega_{C}>0$, at the time $t+\omega_{C}$ transforms into a productively infected cell $I:\left.C_{i i}\right|_{\omega_{C}} \longrightarrow I_{i i}$. 
- The immature virus particle $U$ that arose at time $t$ and did not die during the time interval $\left(t, t+\omega_{U}\right), \omega_{U}>0$, at the time $t+\omega_{U}$ transforms into virion $V:\left.U_{i i}\right|_{\omega_{U}} \longrightarrow V_{i i}$.

- The cell $K$ which arose at time $t$, at time $t+\omega_{K}, \omega_{K}>0$, transforms into a collection of $n_{E}>0$ cytotoxic T-lymphocytes $E:\left.K_{i i}\right|_{\omega_{K}} \longrightarrow n_{E} E_{i i}$.

- Transformation of the cells $T_{i i}, I_{i i}, E_{i i}$ and virions $V_{i i}$ into $T_{i j}, I_{i j}, E_{i j}, V_{i j}$ (start of cell and virion transfer) with rates $\beta_{i j}^{(T)}=q_{i j}^{(T)} \lambda_{i i}^{(T)}, \beta_{i j}^{(I)}=q_{i j}^{(I)} \lambda_{i i}^{(I)}, \beta_{i j}^{(E)}=q_{i j}^{(E)} \lambda_{i i}^{(E)}, \beta_{i j}^{(V)}=q_{i j}^{(V)} \lambda_{i i}^{(V)}$ respectively:

$$
T_{i i} \stackrel{\beta_{i j}^{(T)}}{\longrightarrow} T_{i j} ; \quad I_{i i} \stackrel{\beta_{i j}^{(I)}}{\longrightarrow} I_{i j} ; \quad E_{i i} \stackrel{\beta_{i j}^{(E)}}{\longrightarrow} E_{i j} ; \quad V_{i i} \stackrel{\beta_{i j}^{(V)}}{\longrightarrow} V_{i j} ;
$$

where $q_{i j}^{(\cdot)} \geqslant 0$ is probability that the transition of cells and virions will occur from vertex $N_{i}$ to vertex $N_{j}$ along edge $N_{i j} ; \lambda_{i i}^{(\cdot)}>0$ is rate at which cells and virions begin to transition from vertex $N_{i}$ to vertex $N_{j}$ along edge $N_{i j} ; 1 \leqslant i, j \leqslant n, i \neq j$.

The assumptions made are shown in figure 2.

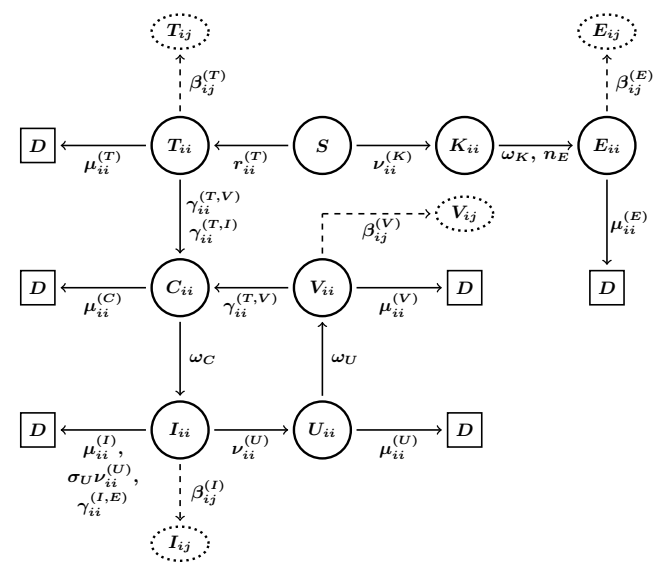

Figure 2. Scheme of the model for a single lymph node $N_{i}$

Let us introduce a set of assumptions that determine the movement of cells $T, I, E$ and virions $V$ along the edges of the graph $G$ :

- The cells $C, K$ and viral particles $U$ don't move along the edges (lymphatic vessels) of the graph $G$.

- Interactions of cells and virions in edges of $G$ is absent.

- Natural death of cells $T_{i j}, I_{i j}, E_{i j}$ and virions $V_{i j}$ :

$$
T_{i j} \stackrel{\mu_{i j}^{(T)}}{\longrightarrow} D ; \quad I_{i j} \stackrel{\mu_{i j}^{(I)}}{\longrightarrow} D ; \quad E_{i j} \stackrel{\mu_{i j}^{(E)}}{\longrightarrow} D ; \quad V_{i j} \stackrel{\mu_{i j}^{(V)}}{\longrightarrow} D .
$$

- The cells $T_{i j}, I_{i j}, E_{i j}$ and virions $V_{i j}$ that started transition from vertex $N_{i}$ to vertex $N_{j}$ along edge $N_{i j}$ at the moment $t$ and didn't die during the transition, at the time

$$
\begin{array}{ll}
\eta_{i j}^{(T)}(t)=t+\Delta_{i j}^{(T)}(t) ; & \eta_{i j}^{(I)}(t)=t+\Delta_{i j}^{(I)}(t) ; \\
\eta_{i j}^{(E)}(t)=t+\Delta_{i j}^{(E)}(t) ; & \eta_{i j}^{(V)}(t)=t+\Delta_{i j}^{(V)}(t) ;
\end{array}
$$

reaches the vertex $N_{j}$ and transforms into $T_{j j}, I_{j j}, E_{j j}, V_{j j}$ respectively. 
The above assumptions have the following probabilistic interpretation. Fix the indexes $1 \leqslant i, j \leqslant n, i \neq j$. Assume, that $h \rightarrow+0$.

The expression $S \stackrel{r_{i i}^{(T)}}{\longrightarrow} S+T_{i i}$ means that over a period of time $(t ; t+h)$, with probability $r_{i i}^{(T)} h+o(h)$ to the vertex $N_{i}$ from $S$ arrives one cell $T_{i i}$, and with probability $1-r_{i i}^{(T)} h+o(h)$ cell entry does not occur.

The expression $T_{i i} \stackrel{\mu_{i i}^{(T)}}{\longrightarrow} D$ means that over a period of time $(t ; t+h)$, with probability $\mu_{i i}^{(T)} h+o(h)$ one cell $T_{i i}$ dies, and with probability $1-\mu_{i i}^{(T)} h+o(h)$ does not die.

The expression $T_{i i}+V_{i i} \stackrel{\gamma_{i i}^{(T, V)}}{\longrightarrow} C_{i i}$ means that over a period of time $(t ; t+h)$, with probability $\gamma_{i i}^{(T, V)} h+o(h)$ one $T_{i i}$ cell interacts with one virion $V$ and with probability $1-\gamma_{i i}^{(T, V)} h+o(h)$ such interaction does not occur.

The expression $T_{i i} \stackrel{\beta_{i j}^{(T)}}{\longrightarrow} T_{i j}$ means that over a period of time $(t ; t+h)$, with probability $\beta_{i j}^{(T)} h+o(h)$ one $T_{i i}$ cell turns into one $T_{i j}$ cell, and with probability $1-\beta_{i j}^{(T)} h+o(h)$ does not turn.

The expression $T_{i j} \stackrel{\mu_{i j}^{(T)}}{\longrightarrow} D$ means that over a period of time $(t ; t+h)$, with probability $\mu_{i j}^{(T)} h+o(h)$ one cell $T_{i j}$ dies, and with probability $1-\mu_{i j}^{(T)} h+o(h)$ does not die.

Other expressions have a similar meaning. Denote

$$
\begin{gathered}
X_{i i}(t)=\left(T_{i i}(t), C_{i i}(t), I_{i i}(t), U_{i i}(t), V_{i i}(t), K_{i i}(t), E_{i i}(t)\right), \\
X_{i j}(t)=\left(T_{i j}(t), C_{i j}(t), I_{i j}(t), U_{i j}(t), V_{i j}(t), K_{i j}(t), E_{i j}(t)\right)
\end{gathered}
$$

vectors containing the number of system components at the vertex $N_{i}$ and at the edge $N_{i j}$ respectively (for each fixed $t$ the components of the vectors are non-negative integer random variables). Note that in this model $C_{i j}(t)=U_{i j}(t)=K_{i j}(t)=0$ for each $t \geqslant 0, i \neq j$. To account for the stages of development of cells and viral particles, we introduce random point-distributions. Random point-distributions contain information about the moments of the appearance of maturing cells and particles, as well as the moments of their transformation into the corresponding cells and particles. For instance, random point-distribution, using to describe $C_{i i}$ cells, has the structure

$$
\begin{gathered}
\Omega_{i i}^{(C)}(t)=\left\{\tau_{i i}^{(C 1)}+\omega_{C}, \ldots, \tau_{i i}^{(C k)}+\omega_{C}, \ldots, \tau_{i i}^{\left(C C_{i i}(t)\right)}+\omega_{C}\right\}, \\
\tau_{i i}^{(C 1)}<\cdots<\tau_{i i}^{(C k)}<\cdots<\tau_{i i}^{\left(C C_{i i}(t)\right)} \leqslant t, \\
\tau_{i i}^{(C k)}+\omega_{C}>t, \quad 1 \leqslant k \leqslant C_{i i}(t), 1 \leqslant i \leqslant n,
\end{gathered}
$$

where $\tau_{i i}^{(C k)}$ is the moment of the appearance of another infected cell $C ; \tau_{i i}^{(C k)}+\omega_{C}$ is the moment of transformation of an infected cell into a productively infected cell $I$ (without taking into account death). If $C_{i i}(t)=0$ for some $t$, then $\Omega_{i i}^{(C)}(t)=\emptyset$. For particles $U_{i i}$ and cells $K_{i i}$ random point-distributions are constructed similarly.

To describe a population of cells $T_{i j}, I_{i j}, E_{i j}$ and virions $V_{i j}$ we also use random pointdistributions reflecting the movement of particles from vertex $N_{i}$ to vertex $N_{j}, i \neq j$. For instance, random point-distribution, using to describe $T_{i j}$ cells, has the structure

$$
\begin{gathered}
\Omega_{i j}^{(T)}(t)=\left\{\eta_{i j}^{(T)}\left(\tau_{i j, 1}^{(T)}\right) ; \ldots ; \eta_{i j}^{(T)}\left(\tau_{i j, k}^{(T)}\right) ; \ldots ; \eta_{i j}^{(T)}\left(\tau_{i j, \zeta(t)}^{(T)}\right)\right\}, \\
\tau_{i j, 1}^{(T)}<\cdots<\tau_{i j, \zeta(t)}^{(T)} \leqslant t<\eta_{i j}^{(T)}\left(\tau_{i j, 1}^{(T)}\right)<\cdots<\eta_{i j}^{(T)}\left(\tau_{i j, \zeta(t)}^{(T)}\right),
\end{gathered}
$$

where $\tau_{i j, k}^{(T)}$ is the moment of the beginning of the transition of a certain particle $T_{i j}$ from vertex $N_{i}$ to vertex $N_{j}$ along the edge $N_{i j}$; and $\eta_{i j}^{(T)}\left(\tau_{i j, k}^{(T)}\right)=\tau_{i j, k}^{(T)}+\Delta_{i j}^{(T)}\left(\tau_{i j, k}^{(T)}\right)$ is the moment 
of completion of the transition provided that the specified particle does not die during the transition. If $T_{i j}(t)=0$ for some $t$, then $\Omega_{i j}^{(T)}(t)=\emptyset$. For $I_{i j}, E_{i j}$ and $V_{i j}$ random pointdistributions are constructed similarly.

Next, we show the state change of the studied random process at discrete moments of time. Assume $t_{0}=0 ; X_{i j}\left(t_{0}\right) \geqslant 0$ are initial numbers of system components at vertices and edges of graph $G ; \Omega_{i i}^{(C)}\left(t_{0}\right), \Omega_{i i}^{(U)}\left(t_{0}\right), \Omega_{i i}^{(K)}\left(t_{0}\right), \Omega_{i j}^{(T)}\left(t_{0}\right), \Omega_{i j}^{(I)}\left(t_{0}\right), \Omega_{i j}^{(E)}\left(t_{0}\right), \Omega_{i j}^{(V)}\left(t_{0}\right)$ are fixed random point-distributions, $1 \leqslant i, j \leqslant n, i \neq j$.

Fix $t_{m}, X_{i j}\left(t_{m}\right), \Omega_{i i}^{(C)}\left(t_{m}\right), \Omega_{i i}^{(U)}\left(t_{m}\right), \Omega_{i i}^{(K)}\left(t_{m}\right), \Omega_{i j}^{(T)}\left(t_{m}\right), \Omega_{i j}^{(I)}\left(t_{m}\right), \Omega_{i j}^{(E)}\left(t_{m}\right), \Omega_{i j}^{(V)}\left(t_{m}\right)$ for some $m=0,1,2, \ldots$ Let

$$
\begin{gathered}
t_{m+1}=\min \left\{\eta^{(m)}, t_{m}+\psi^{(m)}\right\}, \quad X_{i j}\left(t_{m+1}\right)=X_{i j}\left(t_{m}\right)+\Delta X_{i j}\left(t_{m}\right), \\
\Omega_{i i}^{(C)}\left(t_{m+1}\right)=\Omega_{i i}^{(C)}\left(t_{m}\right) \cup\left\{t_{m+1}+\omega_{C}\right\}, \\
\text { or } \Omega_{i i}^{(C)}\left(t_{m+1}\right)=\Omega_{i i}^{(C)}\left(t_{m}\right) \backslash\left\{\tau_{i i}^{(C 1)}+\omega_{C}\right\} \\
\left(\text { for } \Omega_{i i}^{(U)}\left(t_{m+1}\right), \Omega_{i i}^{(K)}\left(t_{m+1}\right) \text { similarly } \Omega_{i i}^{(C)}\left(t_{m+1}\right)\right) ; \\
\Omega_{i j}^{(T)}\left(t_{m+1}\right)=\Omega_{i j}^{(T)}\left(t_{m}\right) \cup\left\{\eta_{i j}^{(T)}\left(t_{m+1}\right)\right\}, \\
\text { or } \Omega_{i j}^{(T)}\left(t_{m+1}\right)=\Omega_{i j}^{(T)}\left(t_{m}\right) \backslash\left\{\eta_{i j}^{(T)}\left(\tau_{i j, 1}^{(T)}\right)\right\} \\
\left.\left.\left(\text { for } \Omega_{i j}^{(I)}\left(t_{m+1}\right)\right), \Omega_{i j}^{(E)}\left(t_{m+1}\right)\right), \Omega_{i j}^{(V)}\left(t_{m+1}\right) \text { similarly } \Omega_{i j}^{(T)}\left(t_{m+1}\right)\right) .
\end{gathered}
$$

Random variable $\eta^{(m)}$ is the nearest to $t_{m}$ time when any of cells $T_{i j}, I_{i j}, E_{i j}$ or virions $V_{i j}$ reach the vertex $N_{j}$ along the edge $N_{i j}$ among all vertices $N_{i}$ and all edges $N_{i j}$ :

$$
\begin{gathered}
\eta^{(m)}=\min \left\{\eta^{(m, T)}, \eta^{(m, I)}, \eta^{(m, E)}, \eta^{(m, V)}\right\}, \\
\eta^{(m, T)}=\min _{(i, j) \in(I, J)_{m, T} \neq \emptyset}\left\{\eta_{i j}^{(T)}\left(\tau_{i j, 1}^{(T)}\right)\right\}, \\
(I, J)_{m, T}=\left\{1 \leqslant i, j \leqslant n, i \neq j: T_{i j}\left(t_{m}\right)>0\right\} .
\end{gathered}
$$

If $(I, J)_{m, T}=\emptyset$, assign $\eta^{(m, T)}=+\infty$. Similarly for $\eta^{(m, I)}, \eta^{(m, E)}$ and $\eta^{(m, V)}$.

Random variable

$$
t_{m}+\psi^{(m)}=\min _{1 \leqslant i \leqslant n}\left\{\tau_{i i}^{(C 1)}+\omega_{C}, \tau_{i i}^{(U 1)}+\omega_{U}, \tau_{i i}^{(K 1)}+\omega_{K}, t_{m}+\xi_{i i}\left(t_{m}\right)\right\}
$$

where $\tau_{i i}^{(C 1)}+\omega_{C}, \tau_{i i}^{(U 1)}+\omega_{U}, \tau_{i i}^{(K 1)}+\omega_{K}$, are the minimal (first) elements of point-distributions $\Omega_{i i}^{(C)}\left(t_{m}\right), \Omega_{i i}^{(U)}\left(t_{m}\right), \Omega_{i i}^{(K)}\left(t_{m}\right)$ respectively; $\xi_{i i}\left(t_{m}\right)$ is random variable representing the duration of time until one of the events at $N_{i}$ :

- inflow of cell $T$ from $S$ to the vertex $N_{i}$;

- death of cells or viral particles at the vertex $N_{i}$;

- interaction of cells and viral particles at the vertex $N_{i}$;

- beginning of the transition of cells and viral particles from vertex $N_{i}$ to vertex $N_{j}$ along the edge $N_{i j}$.

\section{Special case of the model}

Without taking into account the transfer of cells and virions, the stochastic model includes the trivial special case. Let $C_{i i}(0)=I_{i i}(0)=U_{i i}(0)=V_{i i}(0)=K_{i i}(0)=E_{i i}(0)=0,1 \leqslant i \leqslant n$. 
Denote by $\mathbf{E} X_{i i}(t)=\left(\mathbf{E} T_{i i}(t), \mathbf{E} C_{i i}(t), \mathbf{E} I_{i i}(t), \mathbf{E} U_{i i}(t), \mathbf{E} V_{i i}(t), \mathbf{E} K_{i i}(t), \mathbf{E} E_{i i}(t)\right)$ mathematical expectation of the $X_{i i}(t)$. Using the postulates of the random birth and death process, we can derive the differential equation for the mathematical expectation $T^{(i)}(t)=\mathbf{E} T_{i i}(t)$ :

$$
\frac{d T^{(i)}(t)}{d t}=r_{i i}^{(T)}-\mu_{i i}^{(T)} T^{(i)}(t), \quad t \geqslant 0 ; \quad T^{(i)}(0)=\mathbf{E} T_{i i}(0)
$$

The other components of vector $\mathbf{E} X_{i i}(t)$ are identically zero. One can see, that

$$
T^{(i)}(t) \longrightarrow T_{i i}^{*}=r_{i i}^{(T)} / \mu_{i i}^{(T)}, \quad t \rightarrow+\infty
$$

Following [6], we introduce the quotient basic reproductive number for a single lymph node $N_{i}$ :

$$
R_{i}^{(0)}=\frac{\gamma_{i i}^{(T, I)} T_{i i}^{*} e^{-\mu_{i i}^{(C)} \omega_{C}}}{\mu_{i i}^{(I)}+\sigma_{U} v_{i i}^{(U)}}+\frac{v_{i i}^{(U)} \gamma_{i i}^{(T, V)} T_{i i}^{*} e^{-\left(\mu_{i i}^{(C)} \omega_{C}+\mu_{i i}^{(U)} \omega_{U}\right)}}{\left(\mu_{i i}^{(I)}+\sigma_{U} v_{i i}^{(U)}\right)\left(\mu_{i i}^{(V)}+\gamma_{i i}^{(T, V)} T_{i i}^{*}\right)}
$$

The results of computational experiments presented at [6] showed that for $R_{i}^{(0)}<1$

$$
\mathbf{E} X_{i i}(t) \longrightarrow\left(T_{i i}^{*}, 0,0,0,0,0,0\right), \quad t \rightarrow+\infty
$$

\section{Computational experiments}

The purpose of computational experiments is to demonstrate the dynamics of mathematical expectations of variables depending on the variation of values of some model parameters. Number of vertices $n=5$, structure of the graph $G$ is presented in figure 1 . The values of the model parameters were chosen so that $R_{i}^{(0)}<1$ for all vertices or $R_{i}^{(0)}>1$ for at least one of the vertices. The simulation was carried out using the Monte-Carlo method on the time interval $[0 ; W]$. The number of independent realizations of the studied random process is $N=1000$. Components of mathematical expectation $\mathbf{E} X_{i i}(t)$ were estimated by standard formulas of mathematical statistics. We used point $\bar{X}_{i i}(t)$ and interval $\bar{X}_{i i}(t) \pm 1.96 \sigma_{X_{i i}(t)}$ estimates at a confidence level $P=0.95$. For each of two experiments the maximum value of $\sigma_{X_{i i}(t)}$ for the components $\mathbf{E} X_{i i}(t)$ does not exceed 1.62. For computational experiments we used a reference set of parameters, the numerical values of which were selected from the articles [2- 
$4,8,11,12]$ :

$$
\begin{aligned}
r_{11}^{(T)} & =103, \quad r_{22}^{(T)}=90, \quad r_{33}^{(T)}=95, \quad r_{44}^{(T)}=97, \quad r_{55}^{(T)}=80 ; \\
\mu_{i j}^{(T)} & =\mu_{i j}^{(I)}=0.01, \quad 1 \leqslant i, j \leqslant 5 ; \\
\mu_{i i}^{(C)} & =0.01, \quad \mu_{i j}^{(C)}=0, \quad 1 \leqslant i, j \leqslant 5, \quad i \neq j ; \\
\mu_{i i}^{(U)} & =3, \quad \mu_{i j}^{(U)}=0, \quad 1 \leqslant i, j \leqslant 5, \quad i \neq j ; \\
\mu_{i j}^{(V)} & =3, \quad \mu_{i j}^{(E)}=0.43, \quad 1 \leqslant i, j \leqslant 5 ; \\
\gamma_{11}^{(T, V)} & =\gamma_{22}^{(T, V)}=\gamma_{33}^{(T, V)}=6.5 \cdot 10^{-7}, \quad \gamma_{44}^{(T, V)}=4 \cdot 10^{-7}, \\
\gamma_{55}^{(T, V)} & =5.5 \cdot 10^{-7}, \quad \gamma_{i j}^{(T, V)}=0, \quad 1 \leqslant i, j \leqslant 5, \quad i \neq j ; \\
\gamma_{11}^{(T, I)} & =\gamma_{22}^{(T, I)}=\gamma_{44}^{(T, I)}=5 \cdot 10^{-6}, \quad \gamma_{33}^{(T, I)}=10^{-7}, \\
\gamma_{55}^{(T, I)} & =10^{-9}, \quad \gamma_{i j}^{(T, I)}=0, \quad 1 \leqslant i, j \leqslant 5, \quad i \neq j ; \\
\gamma_{i i}^{(I, E)} & =3.7 \cdot 10^{-4}, \quad \gamma_{i j}^{(I, E)}=0, \quad 1 \leqslant i, j \leqslant 5, \quad i \neq j ; \\
v_{11}^{(U)} & =v_{22}^{(U)}=v_{33}^{(U)}=v_{44}^{(U)}=110, \quad v_{55}^{(U)}=200, \\
v_{i j}^{(U)} & =0, \quad 1 \leqslant i, j \leqslant 5, \quad i \neq j ; \\
v_{i i}^{(K)} & =0.05, \quad v_{i j}^{(K)}=0, \quad 1 \leqslant i, j \leqslant 5, \quad i \neq j ; \quad\left(\text { day }^{-1}\right) ; \\
\omega_{C} & =0.2, \quad \omega_{U}=0.021, \quad \omega_{K}=1 ; \quad(\text { day); } \\
\sigma_{U} & =2.618 \cdot 10^{-3}, \quad n_{E}=10 .
\end{aligned}
$$

Parameters of transfer of cells and virions between nodes:

$$
\begin{aligned}
& \lambda_{11}^{(T)}=\lambda_{11}^{(I)}=\lambda_{11}^{(V)}=\lambda_{11}^{(E)}=0.25, \\
& \lambda_{22}^{(T)}=\lambda_{22}^{(I)}=\lambda_{22}^{(V)}=\lambda_{22}^{(E)}=0.45, \\
& \lambda_{33}^{(T)}=\lambda_{33}^{(I)}=\lambda_{33}^{(V)}=\lambda_{33}^{(E)}=0.08, \\
& \lambda_{44}^{(T)}=\lambda_{44}^{(I)}=\lambda_{44}^{(V)}=\lambda_{44}^{(E)}=0.06, \\
& \lambda_{55}^{(T)}=\lambda_{55}^{(I)}=\lambda_{55}^{(V)}=\lambda_{55}^{(E)}=0.37 ; \quad\left(\text { day }^{-1}\right) \text {; } \\
& Q^{(T)}=\left\|q_{i j}^{(T)}\right\|=\left(\begin{array}{ccccc}
0 & 0.4 & 0.6 & 0 & 0 \\
0 & 0 & 0 & 0.444 & 0.556 \\
0 & 0 & 0 & 0 & 1 \\
0 & 0 & 1 & 0 & 0 \\
0.676 & 0 & 0 & 0.324 & 0
\end{array}\right) \\
& Q^{(I)}=Q^{(V)}=Q^{(E)}=Q^{(T)} \text {; } \\
& \Delta_{i j}^{(T)}(t)=0.083+0.1 \cos (t)+0.2 \cos (2 t)+ \\
& +0.05 \cos (3 t)+0.25 \cos (0.5 t) ; \quad \text { (day); } \\
& \Delta_{i j}^{(I)}(t)=\Delta_{i j}^{(V)}(t)=\Delta_{i j}^{(E)}(t)=\Delta_{i j}^{(T)}(t), 1 \leqslant i, j \leqslant 5, i \neq j .
\end{aligned}
$$

The initial conditions for all experiments:

$$
\begin{gathered}
C_{i j}(0)=I_{i j}(0)=U_{i j}(0)=K_{i j}(0)=E_{i j}(0)=0, \\
T_{i i}(0)=r_{i i}^{(T)} / \mu_{i i}^{(T)}, \quad T_{i j}(0)=0, \quad 1 \leqslant i, j \leqslant 5, i \neq j .
\end{gathered}
$$


Experiment 1. The reference set of parameters was used; the quotient basic reproductive numbers are as follows:

$$
\begin{gathered}
R_{1}^{(0)}=0.94, \quad R_{2}^{(0)}=0.82, \quad R_{3}^{(0)}=0.71, \\
R_{4}^{(0)}=0.61, \quad R_{5}^{(0)}=0.51 .
\end{gathered}
$$

On the figures 3, 4 dynamics of the point estimates $T^{(i)}(t)$ without HIV-1 infection, i.e. $V_{i j}(0)=0$, and with HIV-1 infection, i.e. $V_{i i}(0)=10^{4}, V_{i j}(0)=0,1 \leqslant i, j \leqslant 5, i \neq j$, respectively is presented. The numbers indicate the node numbers.

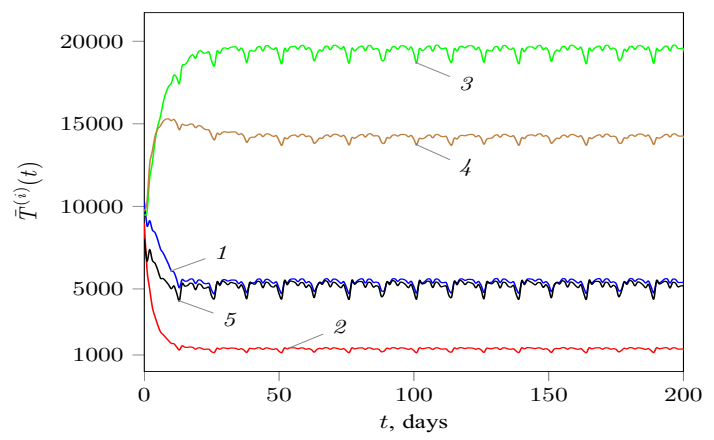

Figure 3. Dynamics of point estimates $T^{(i)}(t)$ without HIV-1 infection at experiment 1.

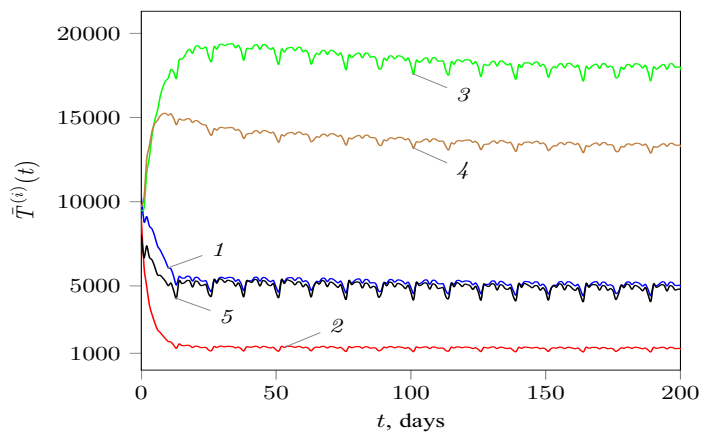

Figure 4. Dynamics of point estimates $T^{(i)}(t)$ with HIV-1 infection at experiment 1.

As can be seen from the figures 3, 4, the number of T-cells decreases slightly in the third and fourth nodes. Let $I(t)=\sum_{i=1}^{5} \sum_{j=1}^{5} \mathbf{E} I_{i j}(t), V(t)=\sum_{i=1}^{5} \sum_{j=1}^{5} \mathbf{E} V_{i j}(t)$. The figures 5, 6 show the dynamics of the point estimates $I(t)$ and $V(t)$ respectively for $V_{i i}(0)=10^{4}, V_{i j}(0)=0$, $1 \leqslant i, j \leqslant 5, i \neq j$. In the figures we can see that the numbers of infected cells and virions remains at some positive level for quite a long time.

Experiment 2. The set of parameters from experiment 1 was used, except

$$
\begin{aligned}
r_{11}^{(T)} & =3500 ; \gamma_{11}^{(T, V)}=10^{-7}, \gamma_{44}^{(T, V)}=\gamma_{55}^{(T, V)}=6.5 \cdot 10^{-7} \\
\gamma_{33}^{(T, I)} & =\gamma_{55}^{(T, I)}=5 \cdot 10^{-6} ; \quad v_{55}^{(U)}=110 .
\end{aligned}
$$




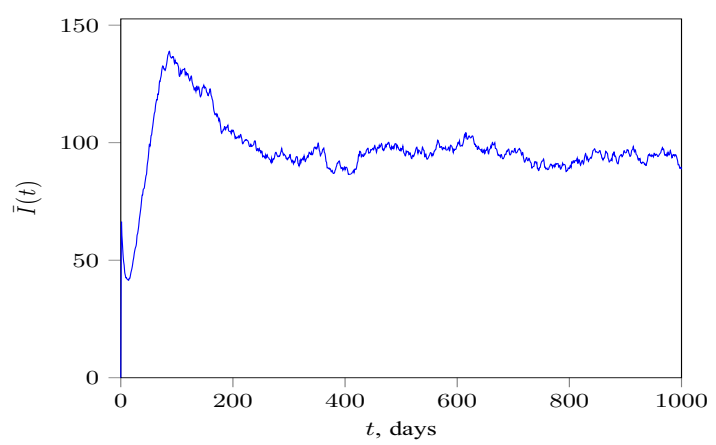

Figure 5. Dynamics of point estimates $I(t)$ at experiment 1.



Figure 6. Dynamics of point estimates $V(t)$ at experiment 1 .

The quotient basic reproductive numbers are as follows:

$$
\begin{gathered}
R_{1}^{(0)}=9.85>1, \quad R_{2}^{(0)}=0.82, \quad R_{3}^{(0)}=0.87, \\
R_{4}^{(0)}=0.89, \quad R_{5}^{(0)}=0.73 .
\end{gathered}
$$

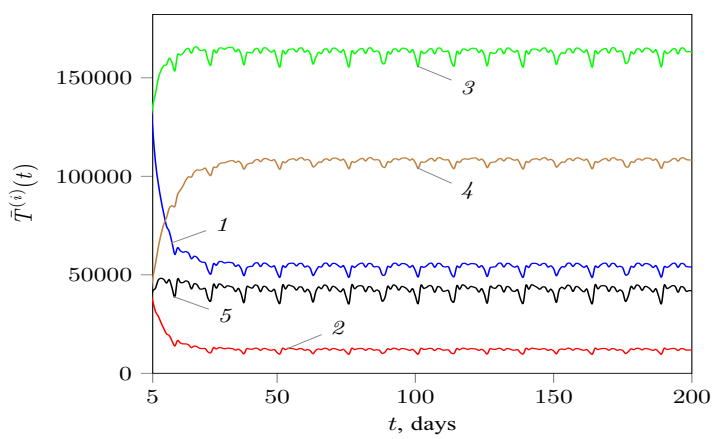

Figure 7. Dynamics of point estimates $T^{(i)}(t)$ without HIV-1 infection at experiment 2. 
On the figures 7, 8 dynamics of the point estimates $T^{(i)}(t)$ without HIV-1 infection, and with HIV-1 infection $\left(V_{i i}(0)=10^{4}, V_{i j}(0)=0,1 \leqslant i, j \leqslant 5, i \neq j\right)$, respectively is presented. As can be seen from the figures 7, 8, number of lymphocytes in HIV-infection is significantly

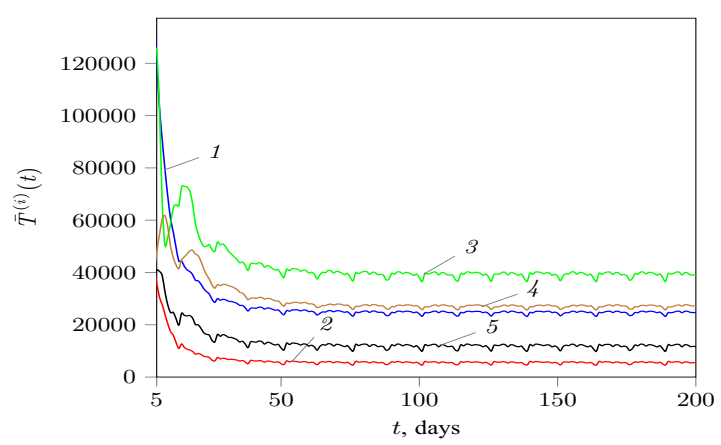

Figure 8. Dynamics of point estimates $T^{(i)}(t)$ with HIV-1 infection at experiment 2.

reduced compared with the absence of HIV-infection. The figures 9, 10 show the dynamics of the point estimates $I(t)$ and $V(t)$ respectively for $V_{i i}(0)=10^{4}, V_{i j}(0)=0,1 \leqslant i, j \leqslant 5$, $i \neq j$, at experiment 2 .

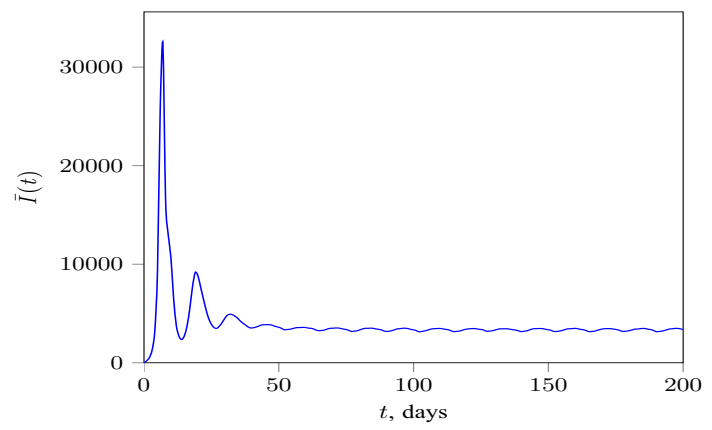

Figure 9. Dynamics of point estimates $I(t)$ at experiment 2.

In the figures 9, 10 we can see that number of infected cells $I$ and virions $V$ after some fluctuations go to some stationary levels. The presence of oscillations is explained by cosines in representation of $\Delta_{i j}^{(I)}(t), \Delta_{i j}^{(V)}(t)$ functions.

\section{Conclusions}

A continuously-discrete compartmental stochastic model of the dynamics of HIV-1 infection has been developed taking into account the interaction of cells and viral particles in a separate lymph node, the maturation of cells and viral particles, as well as the movements of cells and viral particles through the human lymphatic system. An important feature of the developed continuously-discrete stochastic model is the parameterization of the duration of the movement of particles between the compartments using the specified time functions. Further development of the model may be associated with a complication of the processes 




Figure 10. Dynamics of point estimates $V(t)$ at experiment 2.

occurring in the vessels connecting the lymph nodes. In particular, infection of target cells and the production of viral particles by infected cells in the vessels will be considered.

The research was funded by the program of fundamental scientific researches of the Siberian Branch of RAS no. I.1.3.2, project no. 0314-2019-0009.

\section{References}

[1] R. Eftimie, J. J. Gillard, and D. A. Cantrell, Bull.Math. Biol. 78, 2091-2134 (2016).

[2] G. Bocharov, V. Chereshnev, I. Gainova, S. Bazhan, B. Bachmetyev, J. Argilaguet, J. Martinez, and A. Meyerhans, Math. Model. Nat. Phenom. 7 (5), 78-104 (2012).

[3] K. A. Pawelek, S. Liu, F. Pahlevani, and L. Rong, Math. Biosci. 235 (1), 98-109 (2012).

[4] M. Pitchaimani, C. Monica, J. Appl. Math. Comput. 48, 293-319 (2015).

[5] D. S. Taltavull, A. Vieiro, and T. Alarcon, J. Math. Biol. 73, 919-946 (2016).

[6] N. V. Pertsev, B. Yu. Pichugin, and K. K. Loginov, J. Appl. Ind. Math. 13 (1), 103-117 (2019).

[7] I. Gyori, J. Eller, Math. Biosc. 53, 223-247 (1981).

[8] A. S. Mozokhina, S. I. Mukhin, Dif. Eq. 54 (7), 938-944 (2018).

[9] S. Nakaoka, I. Shingo, and K. Sato, J. Math. Biol. 72, 909-938 (2016).

[10] K. K. Loginov, N. V. Pertsev, V. A. Topchii, Math. Biol. Bioinf. 14 (1), 188-203 (2019) (in Russian).

[11] R. M. Tretyakova, G. I. Lobov, G. A. Bocharov. Math. Model. Nat. Phenom. 13 (5), 44-57 (2018).

[12] R. Tretyakova, R. Savinkov, G. Lobov, G. Bocharov. Computation. 6(1), 1-16 (2018). 